\title{
O TDAH: ENTRE AS FUNÇÕES, DISFUNÇÕES E OTIMIZAÇÃO DA ATENÇÃO'
}

\author{
Luciana Vieira Caliman
}

\begin{abstract}
RESUMO. Este artigo analisa a expansão do diagnóstico do Transtorno do Déficit de Atenção/Hiperatividade (TDAH) e suas controvérsias. Desde a década de 80 o TDAH tem sido descrito como uma disfunção neuropsiquiátrica que inicia na infância e continua na vida adulta. O TDAH é visto pelas autoridades médicas internacionais como um problema de ordem pública. Não obstante, apesar de sua legitimidade médica e biológica ser defendida pelo discurso neuropsiquiátrico, a polêmica ao seu redor cresce e se fortalece. Dentre os aspectos críticos do diagnóstico, nosso objetivo principal é analisar as fronteiras frágeis que ele estabelece com as funções e disfunções da atenção e sua otimização. Como diferenciar os sintomas do transtorno da atenção das queixas dos indivíduos que buscam sua otimização? Concluímos o artigo sugerindo questionamentos éticos que devem ser incorporados à clínica diagnóstica e terapêutica do TDAH.
\end{abstract}

Palavras-chave: Transtorno do Déficit de Atenção e Hiperatividade; otimização, neuroética.

\section{THE ADHD: BETWEEN ATTENTIVE FUNCTIONS, DYSFUNCTIONS, AND ENHANCEMENT}

\begin{abstract}
The expansion of the diagnostic category of Attention Deficit Hyperactivity Disorder (ADHD) and its controversies is investigated. Since the 1980s, the ADHD diagnosis has been interpreted as a neuropsychiatric dysfunction that appears in childhood and persists into adulthood. ADHD diagnosis is described by international medical authorities as a public health issue. Although ADHD medical and biological legitimacy has been claimed by neuropsychiatric discourse, the diagnosis has been largely polemical. Among the critical aspects of ADHD diagnosis, current essay discusses the fragile frontiers that ADHD maintains with the functions and dysfunctions of attention and its optimization. How may one differentiate the symptoms of an attention disorder from complaints made by individuals who are looking for attentive optimization? Certain ethic issues that have to be considered in the ADHD clinic are suggested.
\end{abstract}

Key words: Attention Deficit Hyperactivity Disorder, optimization, neuroethic.

\section{EL TDAH: ENTRE LAS FUNCIONES, DISFUNCIONES Y OPTIMIZACIÓN DE LA ATENCIÓN}

\begin{abstract}
RESUMEN. Este artículo analiza la expansión del diagnóstico del Trastorno del Déficit de Atención/Hiperactividad (TDAH) y sus controversias. Desde la década de los 80, el TDAH está siendo descrito como una disfunción neuropsiquiátrica que se inicia en la infancia y sigue en la vida adulta. El TDAH es visto por las autoridades médicas internacionales como un problema de orden público. Pero a pesar de su legitimidad médica y biológica ser defendida por el discurso neuropsiquiátrico, la polémica alrededor crece y se fortalece. De entre los aspectos críticos del diagnóstico, nuestro objetivo principal es analizar las fronteras frágiles que se establece con las funciones y disfunciones de la atención y su optimización. Como diferenciar los síntomas del trastorno de la atención de las quejas de los individuos que buscan su optimización? Concluimos el artículo sugiriendo dudas éticas que deben ser incorporados a la clínica diagnóstica y terapéutica del TDAH..
\end{abstract}

Palabras-clave: Trastorno del Déficit del Atención/Hiperactividad, optimización, neuroética..

\section{O TDAH NA "DÉCADA DO CÉREBRO"}

Em um artigo de 1999, publicado pela Heritage Foundation, Mary Eberstadt descrevia o "cenário
TDAH" na década de 90. De acordo com a autora, desde o final da década 80 e durante os anos noventa, o mundo presenciou uma explosão publicitária sobre o TDAH e a ritalina, naquela época o medicamento

\footnotetext{
Apoio: FAPERJ e DAAD.
}

* Pós-doutoranda do Programa de Pós-Graduação em Psicologia do Instituto de Psicologia da Universidade Federal do Rio de Janeiro-UFRJ. Docente da Faculdade Brasileira UNIVIX, Vitória-ES. 
mais conhecido e utilizado no tratamento do transtorno. Livros sobre o assunto alcançaram a categoria de bestseller, como, por exemplo, Tendência à Distração, de Hallowell e Ratey, publicado em 1994. Entre livros e artigos, a lista estava em crescimento e o adulto TDAH tornava-se parte do público interessado. O TDAH, até então considerado um diagnóstico infantil, passava a ser visto como uma desordem do desenvolvimento que continuava na vida adulta, um quadro crônico incurável.

Em 1994, o quarto volume do Manual Estatístico e Diagnóstico de Doenças Mentais - DSM IV (American Psychiatric Association [APA], 1994) foi publicado e o transtorno do TDAH definitivamente reconhecido como tal. Especialmente nos Estados Unidos da América, a compreensão e o tratamento do TDAH tornaram-se necessidades vinculadas à gestão social e econômica da Nação. Em 2004, o Transtorno do Déficit de Atenção/Hiperatividade foi oficialmente reconhecido, através da Resolução 370 (Estados Unidos da América, 2004), como um dos problemas mais graves e importantes da saúde pública americana. De acordo com as estimativas publicadas nesta resolução, o TDAH abrangia de 3 a $7 \%$ das crianças e adolescentes americanos em idade escolar (2.000.000) e $4 \%$ dos adultos (8.000.000). Devido a esta resolução, o TDAH teve sua entrada nas datas oficiais do país com a proclamação do dia 7 de setembro como o "Dia da Consciência Nacional sobre O TDAH".

As estatísticas americanas sobre o TDAH são também econômicas e baseiam-se na estimativa dos custos médicos e sociais do transtorno. Ao fazer uma revisão bibliográfica sobre o assunto, Matza, Paramone e Prasada (2005) demonstram que um número crescente de pesquisas tem se ocupado da análise das "possíveis" perdas econômicas, aparentemente relacionadas ao TDAH. Além dos gastos com seguro-saúde, medicamentos, consultas e tratamentos, elas analisam o impacto econômico da criminalidade entre indivíduos com TDAH, os custos vinculados ao desemprego ou constantes s de emprego e os relacionados aos frequientes acidentes automobilísticos envolvendo indivíduos com TDAH. Embora baseadas em análises que são, sobretudo, especulativas, estas pesquisas dizem que o TDAH deve ser visto e tratado como um fator de risco, como uma ameaça para os ideais de segurança e de produtividade individual e social do país.

Foi também durante as décadas de 80 e 90 que a interpretação neurobiológica do transtorno tornou-se amplamente aceita. Não é por acaso que, analisando o cenário científico americano, Lakoff (2000) descreve a década de 90, a "década do cérebro", como o auge dos estudos neurológicos sobre o déficit de atenção, financeiramente marcado por um investimento maciço do Instituto Nacional de Saúde Mental (NIMH). Para Barkley (1997a, 1997b, 1998), a autoridade mais conhecida no debate internacional sobre o TDAH, o transtorno era uma disfunção neuropsiquiátrica que afetava o desenvolvimento das "funções executivas" cerebrais, da capacidade de autocontrole, de planejamento e de execução de ações orientadas por objetivos futuros.

Mais do que nunca, os estudos sobre a desordem da atenção e do controle do comportamento e a pesquisa cerebral estavam conectados. A existência do transtorno e a validade do diagnóstico dependiam da descoberta de suas bases biológicas e cerebrais e dos cálculos de seu potencial de risco. No discurso da legitimidade do transtorno, além da comprovação dos males causados pelo TDAH e de seu potencial de risco e, em certo sentido, mais importante do que eles, está o argumento sobre suas causas físicas. Trata-se não apenas de demonstrar que o transtorno "causa" danos ao sujeito, mas que ele, o transtorno, é "causado" por aspectos biológicos, genéticos e cerebrais. Tais suscetibilidades biológicas não são os únicos fatores influentes no desenvolvimento da patologia, mas são, sem dúvida, os mais importantes.

Os dados que fundamentam o discurso da legitimação médica e biológica do TDAH vêm das pesquisas neurológicas e das funções cerebrais, dos estudos feitos com as tecnologias de imagem cerebral e da pesquisa molecular e genética. Através deles, o discurso neuropsiquiátrico dominante diz ser possível demonstrar que o transtorno é real porque, finalmente, seus fatores biológicos foram descobertos e alguns deles se tornaram passíveis de visualização, observação, universalização e comunicação científica. $\mathrm{O}$ argumento pode ser assim resumido: o diagnóstico do TDAH é real porque, em certa medida, é visível e biológico, e descreve uma condição maligna.

Em que pese, não obstante, a ser aclamado como um dos diagnósticos psiquiátricos mais estudados no campo neuropsiquiátrico, o diagnóstico do TDAH também é considerado um dos mais controversos de nossos tempos. Esse diagnóstico tem sido descrito como uma "polêmica" de interesse internacional, que desconhece barreiras culturais e sociais. Os paradoxos em torno do transtorno invadem a mídia mundial, que tem divulgado o TDAH como diagnosis du jour, boutique disorder, psychofad e a Ritalina como "pílula da obediência". Além disso, suas controvérsias são discutidas pelos profissionais mais importantes do campo da neuroética, das ciências humanas e sociais. 
Nos tópicos seguintes, abordamos alguns dos pontos controversos em torno do diagnóstico do Transtorno do Déficit de Atenção/Hiperatividade. Nosso objetivo é estimular o fortalecimento do debate responsável sobre o TDAH nas áreas psicológica, médica e educacional. Não colocamos em questão a realidade, a existência ou a legitimidade do diagnóstico do TDAH. Ao analisar alguns de seus pontos críticos, buscamos contribuir para que, no âmbito das práticas e das políticas de saúde, o TDAH possa ser melhor analisado e seus desafios devidamente considerados.

\section{A "POLÊMICA TDAH": ENTRE AS FUNÇÕES E AS DISFUNÇÕES DA ATENÇÃO E SUA OTIMIZAÇÃO}

Em 2005, em nove países da União Européia, várias conferências intituladas Meetings of Minds foram organizadas com o objetivo de debater questões relevantes sobre a pesquisa cerebral. Representantes das instâncias públicas, legais, científicas, acadêmicas, empresariais e do público em geral reuniram-se com o intuito de formular propostas para a pesquisa cerebral farmacológica, médica, neurológica e ética.

A conferência alemã (Europäische Bürgerkonferenz zur Hirnforschung) teve lugar na cidade de Dresden, nos dias 25, 26 e 27 de novembro de 2005. Entre todos os temas debatidos nessa conferência, as polêmicas em torno do TDAH dominaram a cena. Um dos conferencistas chegou a comentar que era preciso lembrar que a pesquisa cerebral não se resumia à pesquisa sobre o TDAH. No material de propaganda da conferência, a única patologia citada foi o TDAH.

Afinal, quais são as questões que alimentam a polêmica em torno do Transtorno do Déficit de Atenção/Hiperatividade?

Muitos são os aspectos que fazem com que o diagnóstico do TDAH seja descrito como uma "polêmica internacional". O aumento do número de casos identificados em crianças, adolescentes e adultos, a disseminação das prescrições de estimulantes e a proliferação dos processos legais que, com base na evidência do diagnóstico, pleiteavam o direito à educação especial e privilégios no ambiente de trabalho têm contribuído para a disseminação de um clima de suspeita.

Assim como no discurso da legitimação do TDAH, são também os números e estatísticas que alimentam suas controvérsias. Para citar apenas algumas cifras alarmantes a respeito da ritalina, a revista The New York Times, de janeiro de 1999, informava que a produção do medicamento havia aumentado em torno de $700 \%$ desde o início da década, devido ao número de crianças americanas sob medicação. Em 1999, os EUA fizeram uso de 85\% da produção mundial de metilfenidato para propósitos médicos, e é preciso lembrar que este medicamento não é comumente usado no tratamento de outras patologias (com exceção da narcolepsia). O número de consumidores crescia e era especialmente formado pelo público infantil e adolescente.

Um dos dados mais alarmantes era oferecido por Degrandpre (2000), que, comentando a análise de alguns especialistas, dizia que $7 \%$ da população mundial já haviam sido diagnosticados. Em uma análise mais recente, Singh (2005) revelava que estimativas de 2001 diziam que aproximadamente 3\% das crianças americanas em idade escolar estavam tomando algum tipo de medicamento estimulante contra o TDAH. Em relação aos processos legais que, baseados no diagnóstico do TDAH, solicitavam o direito à educação especial, as estatísticas de 2000, analisadas por Gordon e Keiser (1998), revelavam que nos últimos 5 anos este número havia surpreendentemente dobrado e começava a preocupar as organizações públicas.

Na Alemanha, em 2004, um volume inteiro da Revista Cérebro e Mente (Gehirn\&Geist), intitulado "Geração TDAH" (Generation ADHS), foi especialmente dedicado à discussão do TDAH. De acordo com Könneker (2004), os números alemães indicavam que cerca de 500 mil crianças e adolescentes já haviam sido diagnosticadas. No Brasil, não são poucos os dados sobre o diagnóstico do TDAH e a ritalina que alimentam sua polêmica. Revistas e jornais de abrangência nacional, como a Revista Veja, a revista Época e o jornal Folha de São Paulo têm abordado o tema continuamente. Em uma reportagem mais recente da Época, de dezembro de 2006, intitulada "Remédios demais? Os riscos enfrentados por crianças e adolescentes que tomam medicamentos psiquiátricos para tudo - de falta de atenção a mau comportamento", Segatto, Padilla e Frutuoso (2006) revelavam que, nos últimos cinco anos, no Brasil, as vendas da Ritalina haviam triplicado.

Os dados acima revelados fazem parte da polêmica ética, moral, econômica e política criada em torno do TDAH. A discussão de cada um deles demandaria a escrita de outros artigos, mas o panorama traçado nos oferece um pano de fundo que contextualiza nossa discussão. Uma das preocupações do debate bioético e neuroético atual em torno do TDAH diz respeito à separação entre o tratamento das 
patologias da atenção e a otimização das habilidades atentivas, requeridas principalmente pelo espaço ocupacional e escolar. Até onde estamos tratando de uma patologia? Quando estamos buscando a melhora da performance atentiva? Estas questões estão situadas no centro do debate sobre o diagnóstico do TDAH e seu tratamento.

A diferença entre tratamento e otimização foi originalmente proposta no contexto da terapia genética por Sabin e Daniels (1994). O objetivo inicial era separar as intervenções que buscavam corrigir as deficiências do funcionamento genético normal e típico da espécie daquelas destinadas à sua otimização e maximização. Extrapolando a pesquisa genética, o termo enhancement technologies tem sido usado para descrever os tratamentos que visam melhorar a performance, a aparência e o comportamento humano quando essa maximização não é considerada necessária em termos médicos.

Principalmente nos EUA, a difícil distinção entre tratamento e otimização ganhou um colorido extremamente econômico, relacionado à decisão sobre o que se deve ou não ser coberto pelos seguros-saúde. Ao discutir algumas das responsabilidades neuroéticas atuais, Racine e Illes (2006) comentam que, desde ao menos a explosão do uso do Prozac nos EUA, ouvimos falar da proliferação das drogas cosméticas, legitimadas por uma neurologia também cosmética. Ambas estão situadas nos espaços imprecisos estabelecidas entre as estratégias de tratamento e otimização da performance e das (dis)funções cognitivas. Nos tópicos seguintes, analisamos as fronteiras indefinidas que o diagnóstico do TDAH estabelece com as funções e disfunções da atenção e sua otimização.

\section{Um diagnóstico controverso}

Para Gordon e Keiser (1998), as controvérsias em torno do diagnóstico do TDAH nascem primeiramente de sua face interna. Os sintomas que definem o transtorno (desatenção, impulsividade e hiperatividade) são, em menor grau, traços comuns da natureza humana. Todo indivíduo é, em certa medida, um pouco desatento, impulsivo, desorganizado, e nem sempre finaliza as tarefas almejadas, especialmente quando o sujeito em questão é uma criança de 6 ou 7 anos de idade.

Para os teóricos do TDAH do campo neuropsiquiátrico, o que diferencia a patologia da atenção e do autocontrole da normalidade e da "superação positiva" do normal não é uma mudança qualitativa, e sim, temporal e de intensidade. Este é um aspecto extremamente controverso na definição do
TDAH. Para que o diagnóstico seja definido, seus sintomas devem ser quantitativamente anormais. $\mathrm{O}$ que os torna signos da patologia é sua intensidade.

Desde o início da década de 80 , principalmente com a publicação da terceira edição do Manual Estatístico e Diagnóstico de Doenças Mentais (APA, 1980), a psiquiatria tem priorizado a análise quantitativa em relação à qualitativa na definição dos transtornos mentais; mas ao assim fazer, ela obscurece a distinção entre o que ela define por normal, anormal e melhor que normal. Esta mesma psiquiatria diz que o indivíduo com TDAH não se diferencia completamente do sujeito normal, ele apenas está um passo atrás no desenvolvimento de suas capacidades.

De acordo com Barkley (1997a), a criança ou o adulto com TDAH será sempre imatura no desenvolvimento da capacidade de persistência da atenção e controle da ação quando comparada aos indivíduos de sua faixa de desenvolvimento; mas a decisão sobre o que é ser um indivíduo maduro ou imaturo na mestria da atenção e do autocontrole extrapola o campo científico e é, sobretudo, social e moral. Por assim ser, ela será sempre local e circunstancial, não sendo possível traçar linhas divisórias que sejam gerais e aplicáveis para todo e qualquer tipo de sociedade.

As dificuldades em traçar as distinções entre o desatento/hiperativo normal e o anormal são ainda maiores quando se considera a lista de sintomas do TDAH. Em um dos mais preciosos estudos sociológicos sobre o TDAH, Rafalovich (2004) define o transtorno como uma pletora de sintomas diferenciados, desde suas primeiras descrições. Partindo desta definição e de outras que dela se aproximam, os principais críticos do TDAH repetem continuamente que um dos aspectos mais polêmicos deste distúrbio refere-se ao seu perfil de pletora de sintomas. Sua classificação inclui tudo, portanto o TDAH é um transtorno guarda-chuva, que não pode ser realmente visto como uma descrição médica clara e unificada.

A descrição é extensa. O indivíduo com TDAH não é capaz de esperar. Quando envolvido em uma conversa, quebrando todos os códigos de etiqueta, ele repetidamente interrompe a fala do outro ou não ouve o que ele tem a dizer. Ele é incapaz de integrar-se socialmente. Assim, a vida social do indivíduo TDAH está sempre por um fio. Nele, a prudência e a reflexão necessárias à direção das ações mais importantes da vida são prejudicadas. Ele é o indivíduo naturalmente propenso ao risco, uma pessoa que se direciona pela lógica do tudo ou nada, que não mede as consequiências de sua ação. E, para o discurso 
psiquiátrico dominante, esta tendência para o imediato não pensado e não refletido faz com que o produto de seu trabalho seja, na maior parte das vezes, insatisfatório. O comportamento hiperativo também se divide em diferentes signos, desde a manifestação de movimentos corporais excessivos e a incapacidade de permanecer sentado até a agitação interior ou ansiedade constante.

Além disso, o problema da definição do diagnóstico do TDAH se agrava quando outros quadros patológicos descritos na quarta edição do Manual Estatístico e Diagnóstico de Doenças Mentais ou DSM IV (APA, 1994) são considerados. Grande parte das patologias incluídas no manual psiquiátrico americano manifestam um problema de desatenção, de concentração e de impulsividade, e um defeito de inibição.

$\mathrm{O}$ discurso psiquiátrico afirma que para diferenciar um indivíduo sem o transtorno do indivíduo com TDAH são feitas comparações. Suas performances produtivas, sua adaptação social às exigências de seu entorno e sua capacidade de autocontrole são confrontadas. Mas como definir cientificamente esta comparação? A suspeita em torno do diagnóstico do TDAH é também marcada pelo dilema metodológico da objetividade científica. Embora as imagens cerebrais tentem dizer o contrário, a linha que separa o indivíduo TDAH do sujeito normal é tão frágil e tênue que, na clínica e na esfera da vida prática, longe do ambiente laboratorial, ela não pode ser traçada e muito menos visualizada. Apesar dos avanços dos métodos de visualização cerebral, no dia-a-dia da prática diagnóstica eles não revelam muita coisa. Até o momento, nenhum teste ou exame específico e preciso para a "identificação" do TDAH foi definido. Seu diagnóstico continua sendo feito através de um processo misto, que inclui testes psicológicos, história clínica, análise do desempenho escolar e entrevistas com pais e professores.

Quando se trata do adulto, a definição do diagnóstico do TDAH depende da avaliação subjetiva do sujeito afetado. Ele deve avaliar sua história de vida desde a mais tenra infância e nela encontrar traços do TDAH. Além do paciente, outros informantes - como pais, irmãos, cônjuge, colegas de trabalho, advogados e, em certos casos, até mesmo profissionais do trânsito - participam do processo de construção diagnóstica e da reconstrução da narrativa de vida individual. Através do preenchimento de questionários sobre o comportamento da pessoa diagnosticada ou de entrevistas com o médico, a opinião de tais informantes torna-se parte integrante da avaliação do paciente.
Na prática, a construção do diagnóstico do TDAH é melhor descrita como um processo de negociação no qual opiniões diversas devem ser consideradas. Nem sempre estas negociações são pacíficas. Em muitos casos, as opiniões e os interesses das partes implicadas são divergentes: os informantes não estão de acordo entre si ou discordam da auto-análise do paciente, o médico não concorda com a avaliação do paciente e dos informantes e vice-versa. As pessoas podem estar convencidas de que têm a desordem antes de receberem o diagnóstico oficial ou, contrariamente, serem previamente resistentes a ele. Em todo caso, elas também participam ativamente da construção diagnóstica e são profundamente inseridas nesse processo.

Como afirma Berrios (1996), os diagnósticos psicopatológicos são constructos cujas fronteiras e limites dependem, em grande parte, da intenção de seus criadores e das pessoas neles enquadradas, mas entre os primeiros e os segundos nem sempre há acordos. Para os que lidam cotidianamente com o problema do TDAH, os indivíduos diagnosticados, suas famílias ou os profissionais de sua clínica, a pergunta sobre a existência real do transtorno é um prisma composto de muitas partes e sentidos, nem simples nem evidente. Muitos elementos entram em jogo na decisão sobre o que é real, o que não é real, o que é patológico, o que é normal e se o TDAH se inclui em um ou em outro desses reinos.

A distinção entre o sujeito desatento e hiperativo normal, o indivíduo com TDAH e aqueles que buscam a superação das suas capacidades cognitivas surge do envolvimento dos sujeitos implicados na avaliação diagnóstica, bem como das partes interessadas na pesquisa da atenção. Como demonstra Singh (2003, 2004), ela resulta da mistura das expectativas pessoais, sociais, morais e econômicas presentes no processo de constituição do diagnóstico do TDAH. E, para que uma clínica diagnóstica do TDAH seja possível, todas estas expectativas devem ser analisadas, consideradas e problematizadas.

\section{Para além da clínica médica}

$\mathrm{Na}$ distância que separa e aproxima os indivíduos com TDAH e os sujeitos que buscam a otimização da performance atentiva é constituído um espaço polêmico. Nele se alojam também as partes que, de dentro ou de fora do discurso médico, se interessam pelo controle e gestão da atenção e do comportamento. Entre essas partes estão: o mercado farmacológico; as forças armadas; o universo empresarial e sua demanda pelo aumento de produtividade e sucesso; o mercado esportivo; o espaço educacional; as partes diretamente 
implicadas no processo diagnóstico e, de forma particular, o próprio indivíduo com TDAH, com suas demandas e seus dilemas existenciais e sociais.

Assim, foi também durante a década de 90 que o debate sobre os direitos da criança com TDAH aos serviços especiais conquistava novos espaços na legislação americana, através da formulação do ADA (Americans with Disabilities Act of 1990) e do IDEA (The Individuals with Disabilities Education Act of 1997). Aclamado pelas associações americanas de pais e indivíduos TDAH como um passo fundamental na conquista de seus direitos civis, o IDEA visava garantir a assistência financeira federal e local à educação especial de crianças com problemas de aprendizagem. Ser reconhecido como um "indivíduo TDAH" tornava-se um direito civil vinculado a decisão judiciária.

O diagnóstico do TDAH foi legitimado nos campos educacional e legal, mas ao mesmo tempo o uso abusivo de tais ferramentas também alimenta o clima de suspeita em torno do diagnóstico, tornando ainda mais frágeis as linhas que separam a condição mórbida que ele descreve do desempenho normal da atenção e das tentativas de superação de seus limites. Vale lembrar que, no Brasil, já encontramos casos judiciários de pais que reivindicam condições especiais de ensino para os filhos com TDAH, e, em muitos casos, tais condições são também funcionais para crianças não diagnosticadas com TDAH, mas com desempenho educacional "abaixo da média", ou ainda para aquelas que não exploraram em plenitude o seu potencial.

Os avanços psicofarmacológicos também participam da construção do lugar polêmico ocupado pelo diagnóstico do TDAH. A farmacologia do transtorno da atenção e da hiperatividade se confunde e por vezes se identifica com as drogas cosméticas que buscam melhorar a performance cerebral. Estimulantes são drogas que prometem aumentar as capacidades de concentração, de memória e de atenção, necessárias ao desenvolvimento da performance produtiva. Em muitos casos, eles são efetivos tanto em pessoas acometidas pelo TDAH quanto em indivíduos normais insatisfeitos com o seu baixo rendimento profissional e acadêmico. Como comentam Elliot (1999), Singh (2005) e Racine e Illes (2006), pesquisas atuais têm demonstrado que o uso recreativo de metilfenidato, especialmente da ritalina, na otimização da performance acadêmica e profissional tem crescido surpreendentemente.

Em setembro de 2004, a revista The Economist dizia que ao menos 40 novas drogas cosméticas estavam em desenvolvimento e já eram anunciadas.
$\mathrm{Na}$ lista estavam incluídos medicamentos que prometiam ser mais efetivos do que a ritalina e de menor potencial aditivo, como o provigil e o alertec, que, nos EUA, em 2003 alcançaram o número de US\$290 milhões em vendas. Estas drogas se situam nas fronteiras das distinções comentadas, ora pensadas como tecnologias de otimização, ora incluídas na lista das descobertas milagrosas contra patologias específicas como o TDAH.

$\mathrm{Na}$ linha tênue que separa o TDAH das tecnologias de otimização da atenção situamos também a relação histórica entre a pesquisa da atenção e os interesses das Forças Armadas. Taylor (1995) nos mostra como o retorno do interesse pelo estudo da atenção na segunda metade do século XX resultou de uma demanda social e militar do Pós-Guerra. O estudo dos mecanismos atentivos tornava-se uma necessidade prática. $\mathrm{Na}$ Nasa, eletroencefalogramas eram utilizados pelos cientistas na avaliação da capacidade de focalização e vigilância de seus astronautas.

Desde a Segunda Guerra Mundial o uso de anfetaminas pelos pilotos das Forças Aéreas Americanas tem sido uma prática comum e, há décadas, o US Air Force Fatigue Countermeasures Branch tem estudado os efeitos da privação do sono na diminuição dos estados atentivos. Um número cada vez maior de programas de pesquisa destinados ao estudo das farmacologias da atenção tem sido financiado pelas forças militares. Recentemente, o governo francês sugeriu que a Legião Estrangeira fizesse uso de uma forma de modafinil durante algumas de suas operações. Essa mesma droga vem sendo investigada pelas forças militares americanas. $\mathrm{O}$ modafinil criou uma grande polêmica na World AntiDoping Agency que, dez dias antes das olimpíadas de 2004, adicionou o medicamento na lista de substâncias proibidas. Ele também é indicado para o tratamento do TDAH.

Não são poucas as controvérsias a respeito de drogas que oscilam entre serem comparadas à ilícita cocaína, a uma inofensiva taça de café ou a um elixir milagroso, e recomendadas para uma lista de indivíduos que incluem os soldados americanos e franceses, os CEOs das empresas mais famosas do mundo e os indivíduos com TDAH. Se em muitos casos tais controvérsias são excessivamente panfletárias e mal-fundadas, elas também contribuem para a constituição de uma postura mais responsável diante da clínica diagnóstica do TDAH e de sua terapêutica. Além disso, elas também ajudam a problematizar as atuais tecnologias de otimização da atenção, as premissas que as sustentam e suas conseqüências para a vida humana. 


\section{DESAFIOS NEUROÉTICOS}

Novos diagnósticos médicos e psiquiátricos raramente emergem e se legitimam apenas como resultados de descobertas científicas. Eles são um produto de circunstâncias históricas e sociais complexas, internas e externas ao campo médico, e, como comentam Rose $(2000,2003)$ e Novas e Rose (2000), o diagnóstico do TDAH emergiu em um momento histórico no qual as linhas divisórias entre o normal e o patológico foram dissolvidas e transformadas em um contínuo somático.

Neste contínuo, o indivíduo TDAH ocupa um espaço ambíguo. Os modelos com os quais ele dialoga oscilam entre os pólos das celebridades executivas e do marginal criminoso. Um dos resultados da configuração fronteiriça que define o TDAH é que um traço comum, biológico, cerebral e epidemiológico, mas também moral, social e existencial, é criado entre a criança com TDAH, o criminoso, o dependente químico, o deprimido, o motorista imprudente, o indivíduo patologicamente instável, e, por outro lado, o indivíduo bem-sucedido e tenaz em suas decisões, o acadêmico produtivo e o indivíduo que se orienta pelas regras da vida saudável. Vinculando-os está a maior ou menor capacidade de autocontrole e gestão da atenção.

O diagnóstico do TDAH se constituiu no cerne de uma sociedade na qual o indivíduo bem-sucedido, produtivo e feliz é o autogestor atento, consciente, racional e prudente. Nesta economia, não há limites para as exigências de atenção, e, na busca pela sua maximização, todo indivíduo será um pouco desatento. Por outro lado, a psicofarmacologia atual afirma que logo não haverá limites para a intensificação da atenção. A lógica das individualidades somáticas, corrigíveis e manipuláveis nos diz que tudo é possível. O transtorno da atenção e do autocontrole legitimou-se neste contexto, quando as limitações corporais e cerebrais da atenção foram parcialmente eliminadas. Não obstante, é a análise neuroética deste mesmo transtorno que nos impõe o questionamento sobre os limites médicos e éticos das tecnologias de otimização e tratamento da (des)atenção.

Ao analisarem os desafios neuroéticos trazidos pelo desenvolvimento das novas tecnologias de otimização da memória e da atenção, Racine e Illes (2006) identificam ao menos 4 atitudes éticas que, a nosso ver, deveriam ser consideradas pelos profissionais que lidam com o TDAH e incorporadas em sua clínica: 1) o esclarecimento aos pacientes e familiares sobre os limites e as consequiências biológicas, psicológicas e sociais do uso das drogas situadas nas fronteiras entre as estratégias de otimização e tratamento da (des)atenção; 2) no caso da ritalina em particular, a análise da vulnerabilidade das crianças e adolescentes diagnosticados, especialmente no que diz respeito à pressão social, familiar e escolar; 3) a identificação das causas e expectativas individuais e sociais que sustentam a suspeita (ou mesmo o desejo) da existência do diagnóstico; 4) o esclarecimento sobre os usos eticamente "aceitáveis" do tratamento indicado, com o intuito de prevenir possíveis abusos.

A prática diagnóstica e terapêutica do TDAH não é simples, tampouco óbvia. Defender a existência biológica do transtorno e privilegiar a terapêutica medicamentosa não eximem o médico, o psicólogo, o profissional da saúde ou da educação de considerar todos os aspectos, individuais, econômicos, morais e sociais envolvidos em sua clínica. Este é o desafio que o diagnóstico do TDAH impõe aos profissionais da saúde. São estes profissionais que devem alimentar a discussão neuroética em torno do TDAH; são os dilemas levantados no dia-a-dia da clínica do TDAH que podem construir um debate neuroético mais empírico, comprometido com a realidade daqueles envolvidos direta ou indiretamente no lidar com o Transtorno do Déficit de Atenção/Hiperatividade.

$\mathrm{O}$ problema das distinções entre as funções e disfunções da atenção e sua otimização está situado no centro do diagnóstico do TDAH. Ele se refere à complicada distinção médica, ética e moral entre o funcionamento normal e o patológico da atenção e do autocontrole, mas também tangencia as estratégias neurocientíficas atuais de superar o seu estado normal, de otimizá-lo e de ir além de seus limites. O diagnóstico do TDAH constituiu-se no interior deste campo híbrido, ele se alimenta dele ao mesmo tempo em que é por ele fragilizado. Em todo caso, a análise do TDAH como um diagnóstico limítrofe é o desafio neuroético colocado à sua clínica diagnóstica e terapêutica.

\section{REFERÊNCIAS}

American Psychiatric Association - APA (1980). Diagnostic and statistical manual of mental disorders ( $3^{\mathrm{a}}$ ed.). Washington: Author.

American Psychiatric Association - APA (1994). Diagnostic and statistical manual of mental disorders ( $4^{\mathrm{a}} \mathrm{ed}$.). Washington: Author.

Barkley, R. A. (1997a). ADHD and the nature of self-control. London: Guilford. 
Barkley, R. A. (1997b). Behavioral inhibition, sustained attention, and executive functions: Constructing a unifying theory of ADHD. Psychological Bulletin, 121(1), 65-94.

Barkley, R. A. (1998). Developmental course, adult outcome, and clinic-referred ADHD adults. In R. A. Barkley (Ed.), Attention deficit hyperactivity disorder: A handbook for diagnosis and treatment (pp. 186-224). New York: Guilford.

Berrios, G. E. (1996). The history of mental symptoms: Descriptive psychopathology since the nineteenth century. Cambridge: Cambridge.

Degrandpre, R. (2000). Ritalin nation: Rapid-fire-culture and the transformation of human counciousness. New York: W. W. Norton \& Company.

Eberstadt, M. (1999). Why ritalin rules. Policy Review, 94, 1-13. Retrieved in July 20, 2004, from http://www.policyreview.org.

Elliot, C. (1999). A philosophical disease: Bioethics, culture and identity. London: Routledge.

Gordon, M. \& Keiser, S. (1998). Accommodations in higher education under the Americans with disabilities act (ADA): A no-nonsense guide for clinicians, educators, administrators, and lawyers. New York: Guilford.

Hallowell, E. M. \& Ratey, J. J. (1999). Tendência à distração: identificação e gerência do distúrbio do déficit de atenção (DDA) da infância à vida adulta (A. Carvalho, Trad.). Rio de Janeiro: Rocco. (Original publicado em 1994).

Könneker, C. (2004, März 04). Zappelige zeiten. Gehirn\&Geist, p. 3.

Lakoff, A. (2000). Adaptive will: The evolution of attention deficit disorder. Journal of the History of the Behavioral Sciences, 36(2), 149-169.

Matza, L., Paramore, C. \& Prasada, M. (2005). A review of the economic burden of ADHD. Cost effectiveness and resource allocation, 3(5), 1-9. Retrieved in November 11, 2005, from http://www.resource-allocation.com/content/3/1/5.

Novas, C \& Rose, N. (2000). Genetic risk and the birth of the somatic individual. Economy and Society, 29(4), 485-513.

Racine, E. \& Illes, J. (2006). Neuroethical responsibilities. The Canadian journal of neurological sciences, 33, 269-277.

Rafalovich, A. (2004). Framing ADHD children: A critical examination of the History, discourse, and everyday experience of attention deficit/hyperactivity disorder. Latham: Rowman \& Littlefield-Lexington.

Estados Unidos da América (2004). Resolução 370 de 7 de junho de 2004. $108^{\text {th }}$ Congress $2^{\text {nd }}$ Session. Retrieved in August 27, 2007, from http://www.govrecords.org/sres-370-rs-designatingseptember-7-2004-as.html.

Rose, N. (2000). The biology of culpability: Pathological identity and crime control in a biological culture. Theoretical Criminology, 4(1), 5-34.

Rose, N. (2003). The neurochemical self and its anomalies. In A. Doyle (Ed.), Risk and morality (pp. 407-437). Toronto: Toronto.

Sabin, J. \& Daniels, N. (1994). Determining "medical necessity" in mental health practice. Hastings Centre Report, 24(6), 5-13.

Segatto, C., Padilla, I. \& Frutuoso, S. (2006). Remédios demais? Os riscos enfrentados por crianças e adolescentes que tomam medicamentos psiquiátricos para tudo: de falta de atenção a mau comportamento. Revista Época, 446, 108-115.

Singh, I. (2002). Bad boys, good mothers and the "miracle" of Ritalin. Science in context, 15(4), 577-603.

Singh, I. (2003). Boys will be boys: Fathers' perspectives on ADHD symptoms, diagnosis, and drug treatment. Harvard Review of Psychiatry, 11(6), 308-316.

Singh, I. (2004). Doing their jobs: mothering with Ritalin in a culture of mother-blame. Social Science \& Medicine, 59, 11931205.

Singh, I. (2005). Will the "real boy" please behave: Dosing dilemmas for parents of boys with ADHD. The American Journal of Bioethics, 5(3), 34-47.

Report (2004, 16 de setembro). Supercharging the brain. The Economist, pp. 1-5. Retrived in August 27, 2007, from http://www.economist.com/science/tq/displayStory.cfm?story_i $\mathrm{d}=3171454$.

Taylor, E. (1995). Dysfunctions of attention. In J. Cicchetti (Ed.), Developmental psychopathology: Risk, disorder, and adaptation (Vol. 2, pp. 243-273). New York: John Wiley \& Sons.

Recebido em 15/04/2007

Aceito em 15/06/2007

Endereço para correspondência : Luciana Vieira Caliman. Rua Castelo Branco, n. 100, ap. 903, CEP 29100-040, Vila Velha-ES. E-mail: caliman@mpiwg-berlin.mpg.de 